\title{
Heartbeat: Focus on atrial fibrillation
}

Atrial fibrillation is very common; the estimated prevalence increases with age from about $0.1 \%$ in adults under age 55 years to $9 \%$ of those 80 years of age or older. AF is associated with a reduced quality of life due to the irregular heart rate and reduction in cardiac output. More importantly, AF is associated with an increased risk of thromboembolic events, with an annual stroke risk ranging from as low as $<1 \%$ in patients with isolated $\mathrm{AF}$ to as high as $12 \%$ depending on additive risk factors including age over 75 years, female sex, diabetes, hypertension, heart failure, vascular disease or a prior stroke. Stroke risk is even higher in those with AF due to valvular heart disease. Thus, lifelong oral anticoagulant therapy is recommended in most patients to reduce stroke risk.

The presumed pathophysiology of stroke in patients with AF is that stasis of blood flow in the left atrium leads to thrombus formation, most often (90\%) in the left atrial appendage (LAA). Thus, there has been considerable interest in the idea of removing or occluding the LAA to prevent thromboembolic events with the hope of avoiding long-term oral anticoagulant therapy. However, even though LAA occluder devices have been available in Europe for several years and one was recently approved in the USA, this approach remains controversial.

In this issue of Heart Dr López Mínguez and colleagues (see page 877) report 2-year outcomes after LAA occlusion in a consecutive series of 167 patients with nonvalvular AF who had contraindications to long term oral anticoagulation. Device implantation was successful in $94.6 \%$ with procedural complications in $5.38 \%$ of patients. All were continued on clopidogrel for 3-6 months and aspirin for 6-12 after LAA occlusion, unless there were bleeding events. Transesophageal echocardiography at 1 day and at 3, 6 and 12 months after LAA closure showed device thrombus in $8.2 \%$, a small residual atrial septal defect in $11.4 \%$ and an occluder device leak in $8.2 \%$. Major clinical endpoints were an overall mortality of $10.8 \%$ (9.5\% noncardiac), major bleeding

Correspondence to Professor Catherine M Otto, Division of Cardiology, University of Washington, Seattle, WA 98195, USA; cmotto@u.washington.edu in $5.7 \%$ and stroke in $4.4 \%$. (figure 1) The annualized rate of major bleeding and stroke was $2.4 \%$, which was significantly less than expected compared to estimated rates using standard risk scores.

The two editorials that accompany this paper have diametrically opposed points of view about the role of LAA closure in adults with AF. Drs Gafoor, Sievert and Matic (see page 828 ) point out the advantages of LAA occlusion including a low bleeding risk, no issues with patient compliance, lower cost compared to lifetime medication, minimal drug-drug interactions and no need for bridging anticoagulation during future procedures. They conclude: "In short, there is enough safety and clinical efficacy data to make left atrial appendage closure a viable option for stroke prevention in patients with atrial fibrillation".

In contrast, Dr Senoo and Prof Lip (see page 830 ) remind us that not all thromboembolism comes from the left atrial appendage in patients with AF. The pathophysiology of stroke in patients with $\mathrm{AF}$ may also be related to a hypercoagulable

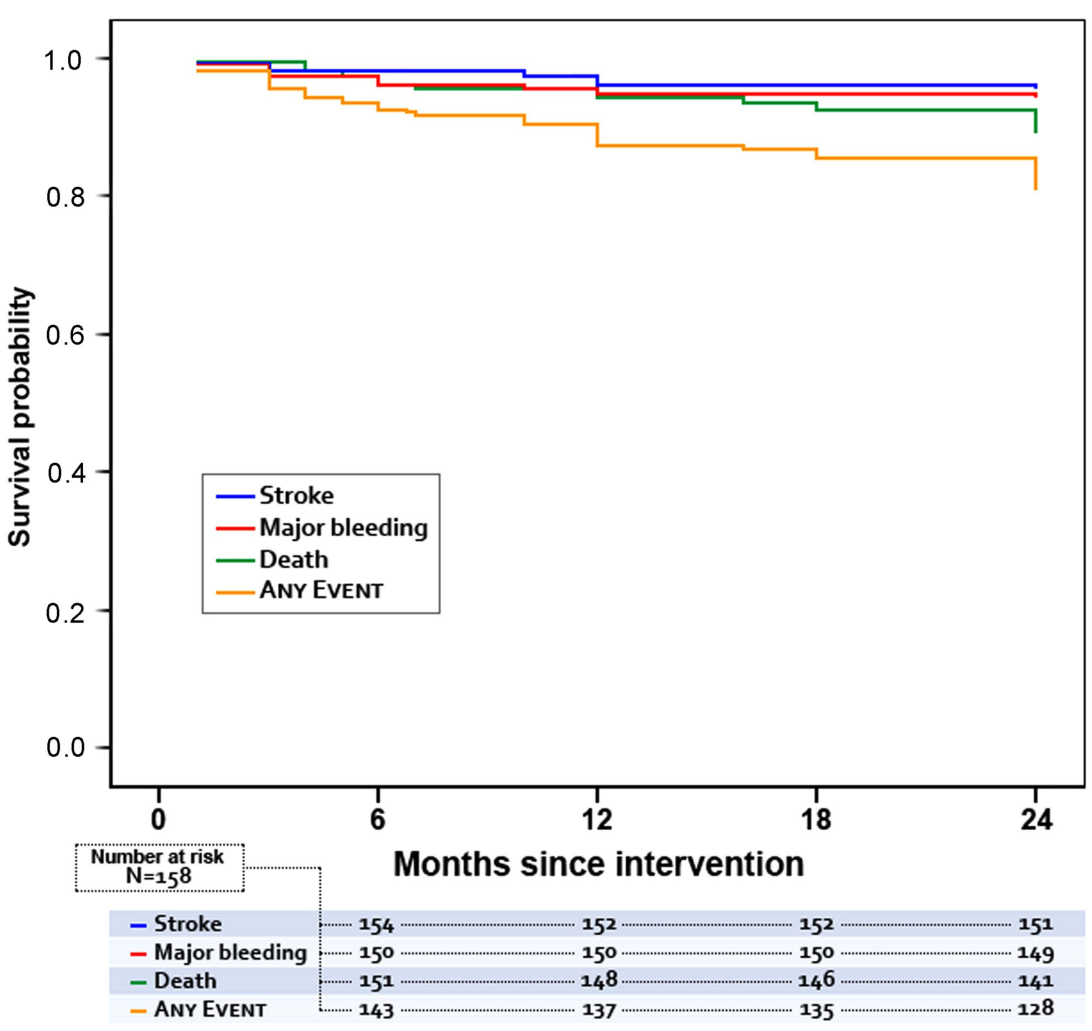

Figure 1 Kaplan-Meier event-free survival curves over 24-month follow-up. state and the presence of other stroke risk factors, including hypertension, diabetes and aortic atherosclerosis. In addition, there currently are no randomized studies comparing LAA occlusion to treatment with newer oral anticoagulants. They conclude: "the current indications of LAA closure should be limited to those patients with AF who are at a very high risk of stroke and have clear contra-indications for any form of anticoagulation or a high risk of bleeding on anticoagulation. Overenthusiasm should be tempered".

A comprehensive review article on LAA occlusion by Drs Alli and Holmes (see page 834) discusses the rationale for this approach, compares transcatheter and surgical closure techniques, summarize the major clinical trials of these devices and provides a practical approach to patient selection and evaluation. (figure 2)

A second review article addresses the question: What can rhythm control therapy contribute to prognosis in atrial fibrillation? (see page 842) The possible benefits and risks of a strategy of rhythm versus rate control are reviewed including 


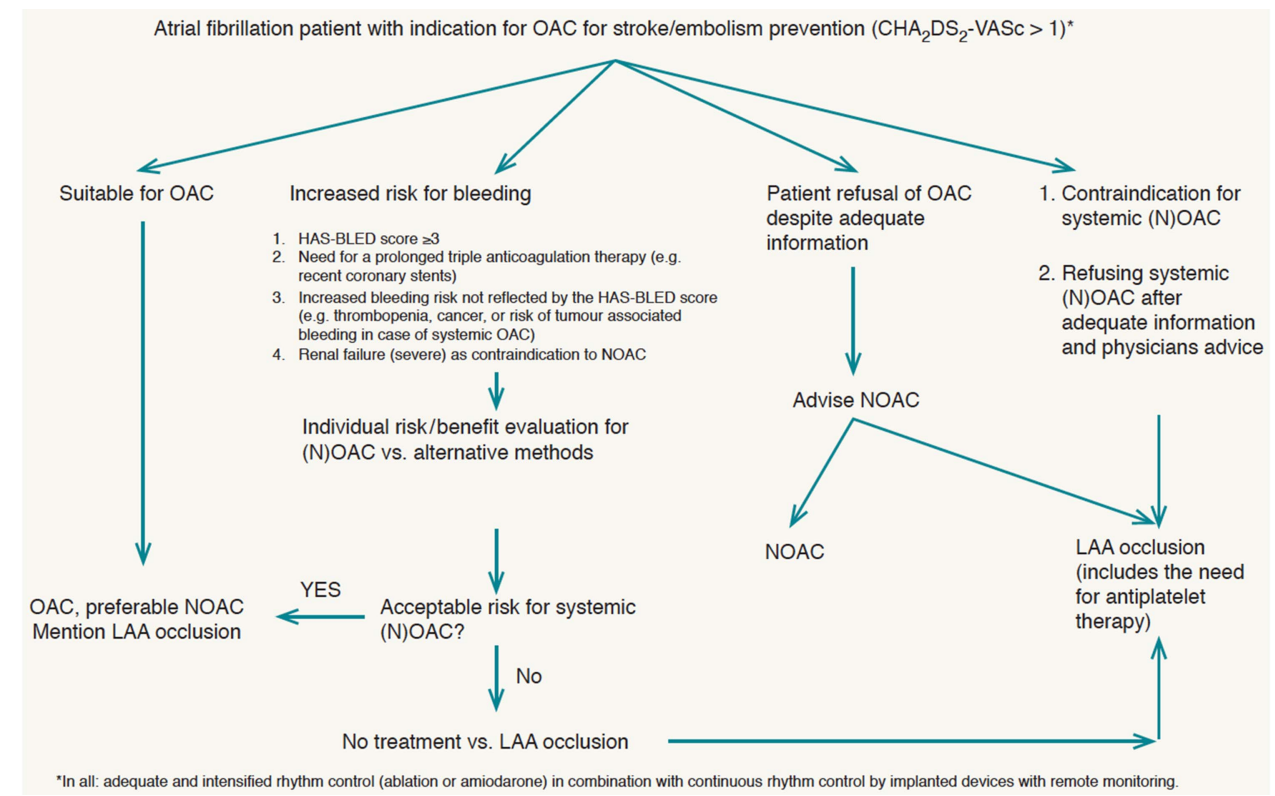

Figure 2 Algorithm for stroke prevention in patients with atrial fibrillation. Recommendations of the European Heart Rhythm Society and the European Association of Percutaneous Cardiovascular Interventions 2014. Reproduced by permission from Meier et al. ${ }^{26}$ (see the reference list of the original paper in this issue). HAS-BLED, Hypertension, Abnormal Renal/Liver Function, Stroke, Bleeding History or Predisposition, Labile INR, Elderly, Drugs/Alcohol Concomitantly; LAA, left atrial appendage; OAC, oral anticoagulants.

the potential toxicity of anti-arrhythmic drugs, the effect of patient age on choice of strategy, the role of heart failure, and whether anticoagulation is needed even with a rhythm control strategy (figure 3 ). Novel options for rhythm control in adults with AF include newer pharmacologic agents and catheter ablation.
However, "at present, anticoagulation is recommended according to the individual thromboembolic risk scores irrespective of the clinical success of rhythm control".

Two other original research articles also focus on $\mathrm{AF}$ in this issue of Heart. In a cohort of over 6000 patients with paroxysmal or persistent AF at baseline, 24\% progressed to permanent AF over a median followup of 18 months (see page 894). The strongest predictors of AF progression were AF on the baseline ECG and older age, while those with a lower heart rate were less likely to progress. These authors conclude: "While a rate or rhythm control strategy is not

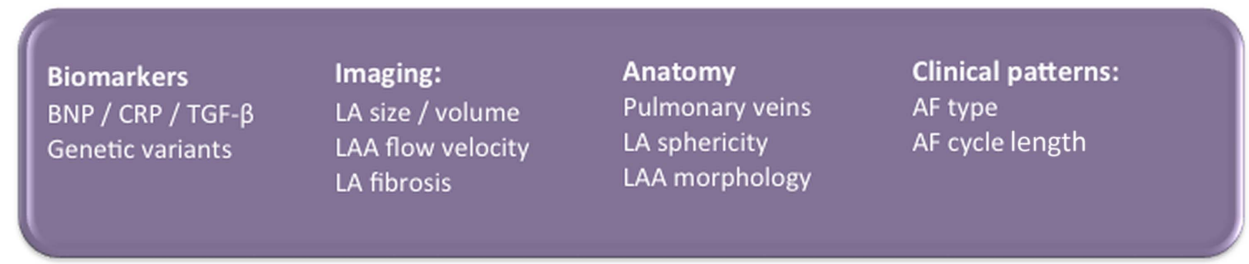

\section{AF substrate}
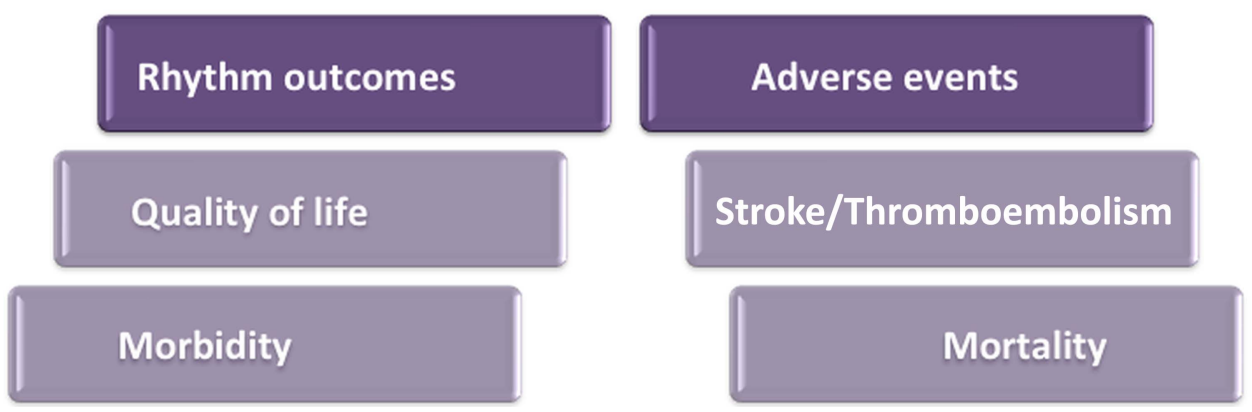

Figure 3 Diagram illustrating factors associated with atrial fibrillation (AF) substrate as well as clinical consequences of the disease condition. BNP, brain natriuretic peptide; CRP, $C$ reactive protein; LA, left atrium; LAA, left atrial appendage; TGF- $\beta$, transforming growth factor- $\beta$. 
independently associated with AF progression, lower heart rate may influence or delay AF progression".

A cross-sectional study with ECG recordings on all consenting patients $(n=647)$ at a University hospital on single day showed a $9.1 \%$ prevalence of $\mathrm{AF}$ (95\% CI $6.9 \%$ to $11.3 \%$ ) with an additional 49 patients having a history of $\mathrm{AF}$ for a total prevalence of $16.8 \%$ (95\% CI $13.9 \%$ to $19.7 \%$ ) across all hospital units (see page 884). Although most patients with AF were at high risk of stroke using standard risk scores, only 51.3\% were receiving appropriate long term anticoagulation emphasizing the need for improved diagnosis and treatment of AF, even in patients receiving regular medical care.

In the Education in Heart article (see page 900) Prof Helge Möllman and colleagues review complications of transcatheter aortic valve implantation with tips on how to avoid and treat these complications. The Key Points box summarizing strategies to reduce complications will be of great value to those training in this increasingly common transcatheter therapy.

Try the Image Challenge (see page 841) which shows an unusual cause of hemodynamic collapse during permanent pacemaker implantation. No doubt it would be best to avoid this complication in the first place, but all who view this image will recognize it quickly should it unfortunately occur in any of their patients.

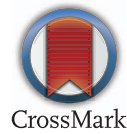

To cite Otto CM. Heart 2015;101:823-825.

Heart 2015:101:823-825.

doi:10.1136/heartjnl-2015-308020 